\title{
RESTOS HUMANOS EN LA COSTA MARINA DEL PARTIDO DE MAR CHIQUITA, PROVINCIA DE BUENOS AIRES
}

\section{HUMAN REMAINS ON THE SEA SHORE OF MAR CHIQUITA DISTRICT, BUENOS AIRES PROVINCE}

\author{
Verónica Aldazabal1* , Emilio Eugenio² y Pamela García Laborde³ \\ ${ }^{I}$ Consejo Nacional de Investigaciones Científicas y Técnicas (CONICET), Instituto Multidisciplinario de Historia y Ciencias Huma- \\ nas - IMIHICIHU. Buenos Aires. Argentina \\ ${ }^{2}$ CONICET, IMIHICIHU. Facultad de Filosofia y Letras. Universidad de Buenos Aires. Buenos Aires. Argentina \\ ${ }^{3}$ CONICET, Laboratorio de Ecología Evolutiva Humana (LEEH-NEIPHPA). Facultad de Ciencias Sociales. Sede Quequén. UNCPBA. \\ Necochea. Argentina
}

PALABRAS CLAVE: restos humanos; análisis bioantropológíco; Holoceno tardío; Buenos Aires

RESUMEN El objetivo del presente trabajo es dar a conocer los resultados de los análisis bioantropológicos realizados sobre los restos óseos humanos que aparecieron de manera fortuita en la costa marina del partido de Mar Chiquita, en el contexto de información disponible para el área. Se plantean las dificultades en las tareas de rescate y se discuten aspectos referidos a conservación y pautas de entierro. Los restos analizados son el primer hallazgo en el sector central de la costa marina de la provincia de Buenos Aires, y comprenden por lo menos tres individuos, uno adulto y dos subadultos. Rev Arg Antrop Biol 22(2), 2020. doi: $10.24215 / 18536387 \mathrm{e} 022$

\section{KEYWORDS humans remains; bioanthropological analysis; Late Holocene; Buenos Aires}

ABSTRACT The aim of the present work is to present the results of the bioanthropological analyses carried out on the human bone remains that appeared fortuitously on the sea shore of Mar Chiquita district, in Buenos Aires province, on the basis of the available information from the area. Difficulties in rescue tasks are addressed, and aspects related to conservation and burial standards are discussed. The remains analyzed are the first finding of this kind on the central marine coast of Buenos Aires province, and comprise at least three individuals, one adult and two subadults. Rev Arg Antrop Biol 22(2), 2020. doi: $10.24215 / 18536387 \mathrm{e} 022$
En Octubre del año 2015 dos docentes que caminaban por la zona de la Albufera de Mar Chiquita, provincia de Buenos Aires, hallaron restos humanos: una mandíbula, un cráneo y varias extremidades. Fueron denunciados a la Policía local, dando intervención a la Fiscalía. Luego de interminables trámites burocráticos, los restos óseos fueron recuperados mediante técnicas de arqueología de rescate, en el término de 5 horas durante los días 4 y 13 de Mayo del año 2016, luego de la intervención de numerosas personas (Equipo Argentino de Antropología Forense - EAAF, Policía, Fiscalía, periodistas). Los relevamientos efectuados permitieron definir la dispersión en un espacio de $3 \mathrm{~m}^{2}$. Se hallaron restos humanos semienterrados, escasos restos faunísticos y líticos. Los restos apoyaban sobre un sedimento negro húmedo, tapado por el médano. El conjunto fue denominado sitio Mar Chiquita 1 (Mch1).

Asociados a este conjunto se recuperaron tres artefactos, dos sobre guijarros costeros y el tercero manufacturado sobre una diáfisis de metapodio de guanaco (Aldazabal y Belotti, 2019; Eugenio y Aldazabal, 2017). Junto a estos hallazgos se recolectaron fragmentos óseos, algunos identificados como faunísticos y otros que no pudieron identificarse, los cuales, debido a su estado fueron excavados y envueltos en papel metálico para evitar mayor fragmentación, ya que no se disponía de la posibilidad de aplicar otros métodos de consolidación. Los estudios realizados permitieron identificar un individuo adulto y dos individuos subadultos,

\footnotetext{
Financiamiento: Proyecto Arqueología en ambientes costeros. OPDS: DI-2019-76. CONICET.

*Correspondencia a: Verónica Aldazabal. IMHICIHU-CONICET. Saavedra 15, $5^{\circ}$. C1083ACA CABA. Argentina. E-mail: varalda2@gmail.com
}

Recibido 4 Noviembre 2019; aceptado 27 Enero 2020

doi: $10.24215 / 18536387 \mathrm{e} 022$ 
además de un conjunto de restos que no pudo asignarse a alguno de los individuos en particular.

A cargo de la Fiscalía se recuperó evidencia que fue seleccionada por el EAAF para ser fechado en el Laboratorio de Tritio y Radiocarbono (Centro de Investigaciones Geológicas - CONICET- Universidad Nacional de La Plata). El fechado radiocarbónico sobre un fragmento de fémur $\left(\mathrm{N}^{\circ} 24974\right)$ dio como resultado $910 \pm 50$ años AP. Este resultado permitió el acceso a la evidencia y al sitio para realizar el rescate de lo restante en el lugar (Fig. 1).

El objetivo del presente trabajo es dar a conocer los resultados de los análisis bioantropológicos realizados sobre los restos óseos humanos en el contexto de información disponible para el área. En las últimas décadas, se han incrementado los hallazgos de restos humanos en la región pampeana intensificando sus estudios a través de diversas líneas de investigación arqueológica. Existe un consenso general entre los investigadores que sostiene una baja demografía y alta movilidad para los grupos de cazadores-recolectores del Holoceno temprano y medio, mientras que, para el Holoceno tardío, se plantea que algunas áreas experimentaron un aumento en el tamaño de la población y en la densidad espacial, con cierta reducción en la movilidad. Sin embargo, en el área de estudio, el sector oriental de la Pampa Deprimida se caracteriza por una ocupación recién a partir del Holoceno tardío y escaso registro bioantropológico. Además éste es el primer registro en ambiente marino.

\section{MATERIAL Y MÉTODOS}

Los restos óseos extraídos comprenden tres conjuntos según el momento de extracción. A medida que se iban recuperando, los restos se preservaban en bolsas de polietileno con sus respectivos rótulos. Todas las piezas fueron limpiadas en seco y con posterioridad a su estudio, los embalajes fueron cambiados a bolsas Ziploc®, libres de ácidos.

El análisis bioarqueológico, comprendió la identificación de los restos óseos humanos versus fauna, la realización de un inventario, el registro fotográfico y la determinación de sexo y edad (en los casos posibles) (García Laborde, 2018).

Para la determinación de sexo y estimación de la edad del individuo adulto se siguieron los métodos propuestos por Buikstra y Ubelaker (1994) y Krenzer (2006). Para la determinación de sexo en subadultos se aplicó el método propuesto por Holger Shutkowski (1993) que enfatiza en la observación del ilion y la mandíbula, aún cuando pueden resultar imprecisos (Guichón y García Laborde, 2014) debido a que estos individuos no alcanzaron claramente las características dimórficas primarias y secundarias que los distinguen. $\mathrm{La}$ edad fue estimada siguiendo los métodos del desarrollo y erupción dental, medición de huesos largos y craneales, empleando los estándares publicados Scheuer y Black (2000) y la fusión ósea (Fazekas y Kósa, 1978; Schaefer, Black, Schaefer y Scheuer, 2009; Scheuer y Black, 2000).

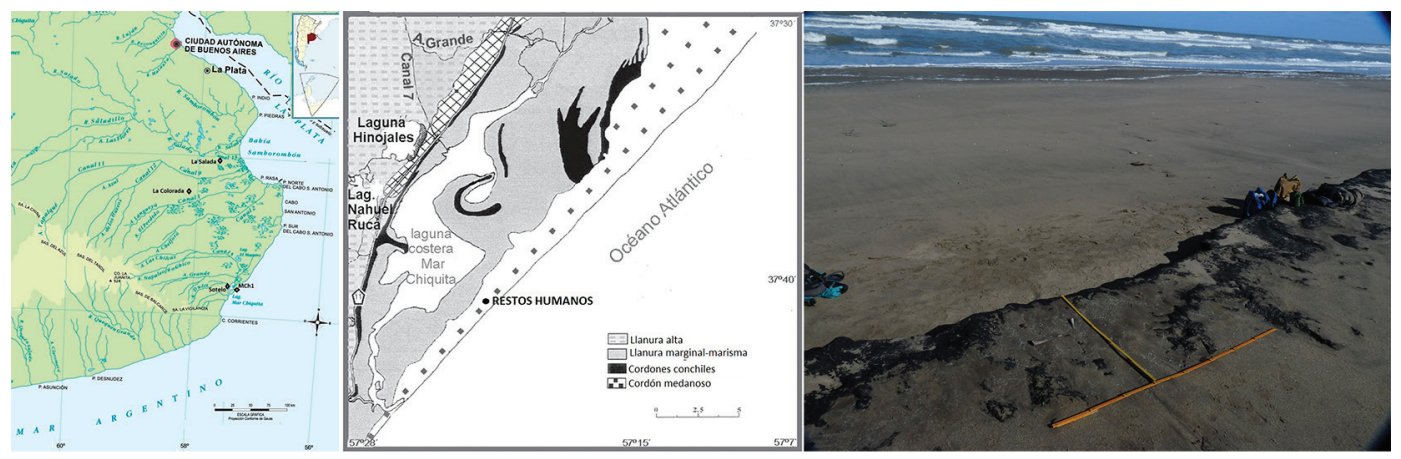

Fig. 1. Localización. Izquierda, ubicación de los sitios con restos humanos. Centro, contexto del sitio MCh. Derecha, detalle del lugar de hallazgo. 
Luego de agrupar el conjunto de restos óseos en base a estos parámetros (edad y sexo) y dimensiones relativas, se pudo calcular el número mínimo de individuos (NMI).

Una pieza dental de cada uno de los individuos identificados fue seleccionada para la determinación de $\mathrm{ADN}$, realizándose una réplica de la pieza dentaria en yeso de alta densidad.

\section{RESULTADOS}

Individuo 1: Se encuentra representado por la mandíbula con su dentadura completa (16 piezas dentales: 4 incisivos, 2 caninos, 4 premolares y 6 molares), fémur derecho e izquierdo, tibia y peroné derechos, radio y cubito derechos y costillas (1 completa y 3 fragmentos). La muestra del fémur derecho seleccionada para realizar el fechado radiocarbónico se asignó a este individuo. Además se extrajo el $1^{\circ}$ Molar Derecho de la mandíbula para realizar estudios de ADN (Universidad de Tennessee, Estados Unidos) aún en proceso. Con los restos presentes se pudo determinar el sexo del individuo, considerándolo masculino a partir de mediciones del fémur (diámetro de la cabeza y circunferencia de la diáfisis), de la tibia (anchura proximal y distal) y de la morfología de la rama mandibular. En relación a la edad, los restos presentes dan cuenta de un individuo adulto por el estado de fusión de los mismos (Fig. 2).

Asociado al individuo 1 fueron recuperados en el sitio algunos fragmentos óseos que no pudieron identificarse, (MCH2- 6, 9, 10, 11, 13 y 14) algunos de ellos inclusive fueron excavados y levantados en bloques de sedimento para evitar mayor fragmentación.
Individuo 2: Los restos óseos que se recuperaron y fueron asignados al Individuo 2, son: cráneo, maxilar (4 incisivos, 3 premolares, 4 molares y 1 molar deciduo), mandíbula (4 incisivos, 2 caninos, 4 premolares, 4 molares y 1 molar deciduo), fémur derecho, ilion izquierdo, escápula derecha, peroné derecho y radio derecho. Se extrajo el $1^{\circ}$ Molar Derecho de la mandíbula para estudios de ADN. A partir de la morfología del cráneo y del método de Schutkowski (2003) que analiza la morfología de la mandíbula y del ilion, se determinó este individuo 2 como de sexo femenino. Con respecto a la edad, fue estimada en un intervalo de entre 10 y 12 años, a partir del método de erupción y desarrollo y las mediciones de algunos de los restos postcraneales, concretamente la longitud del fémur, peroné y radio; la longitud y el ancho del ilion; el ancho de la escapula y el estado de fusión de la epífisis distal del peroné (Fig. 3).

Individuo 3: Los restos asignables al Individuo 3 son: fémur derecho e izquierdo, húmero derecho y las piezas dentales que posiblemente correspondan a dicho individuo son 2 incisivos, 2 premolares, 2 molares y 1 diente deciduo. Se separó un molar para extraer la muestra para estudios de ADN. Los restos óseos presentes no permitieron determinar el sexo del individuo. Con respecto a la edad, la misma fue estimada en un intervalo de entre 6 y 8 años, a partir de la longitud de los fémures y del húmero (Fig. 4).

Restos óseos humanos sin individualizar: Fueron recuperados en el sitio 42 elementos
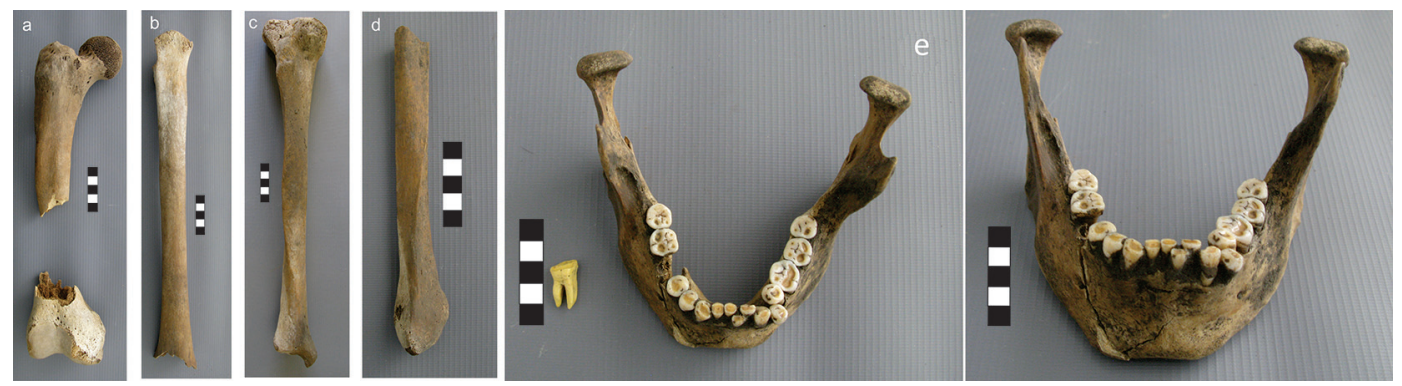

Fig. 2. Individuo 1. a) Fémur derecho. b) Fémur izquierdo. c) Tibia derecha. d) Peroné derecho. e) Mandíbula y réplica dental del $1^{\circ} \mathrm{MD}$ extraido para $\mathrm{ADN}$. 

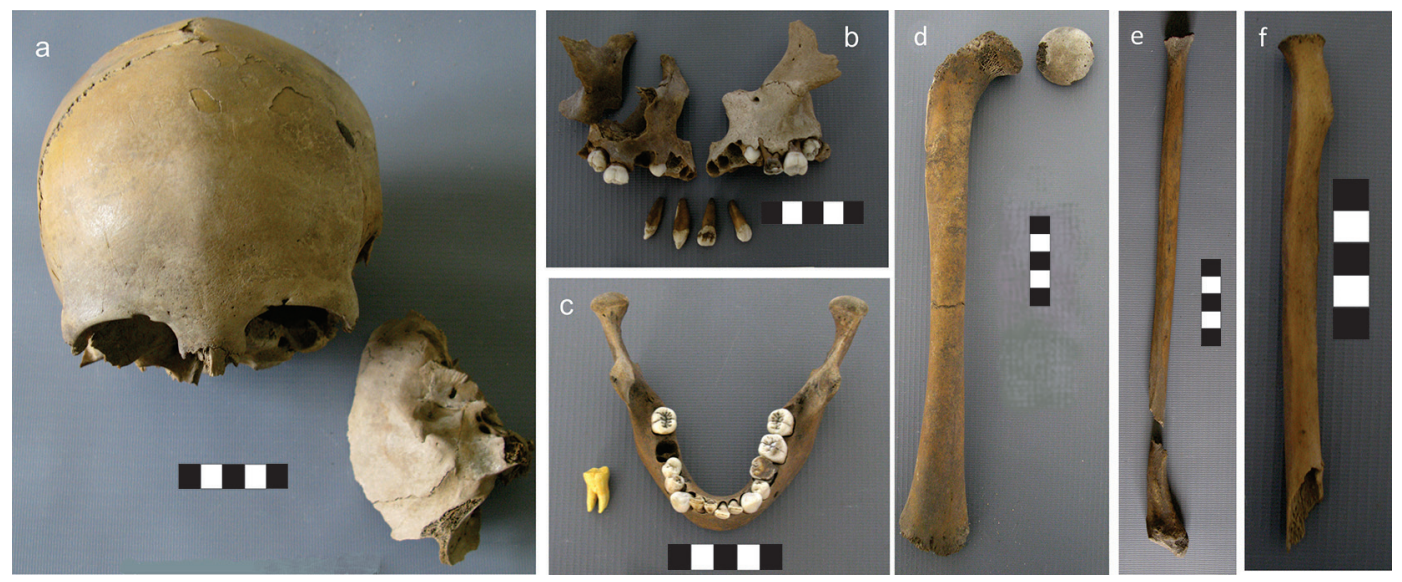

Fig. 3. Individuo 2. a) Cráneo. b) Maxilar. c) Mandíbula, a su izquierda la réplica dental del $1^{\circ} \mathrm{MD}$. d) Fémur derecho. e) Peroné derecho. f) Radio derecho.

óseos, los cuales pudieron ser identificados: fragmentos de calcáneo y astrágalo $(\mathrm{MCH}$ 2-21), proceso coracoides de la escapula derecha (MC-4), 1 metacarpo (sin rótulo), 1 metacarpo (MC-2), $5^{\circ}$ metacarpiano izquierdo (MC-1), $5^{\circ}$ metacarpiano derecho (MCH2-21), navicular (MCH2-21), costillas: 1 izquierda, 2 derechas, 1 fragmento de costilla izquierda, 1 fragmento de costilla derecha ( $\sin$ rótulo), 1 falsa derecha y 1 fragmento indet (MC-4), Vertebras: $1^{\circ}$ sacra; $2^{\circ}, 3^{\circ}$, $4^{\circ}$ y $5^{\circ}$ lumbares; 2 torácicas, 3 fragmentos ( sin rótulo); cervical (MC-2), 2 falanges de mano (MC-3), epífisis distal de tibia derecha (MCH2-21), clavícula derecha (MC-4), epífisis proximal peroné derecho $(\mathrm{MCH} 2-21), 1^{\circ}$ metatarso y 2 cuñas (MC- 6$) ; 3^{\circ}, 4^{\circ}$ y $5^{\circ}$ metatarsianos derechos y fragmento indeterminado (MC-7); $3^{\circ}$ y $5^{\circ}$ metatarsianos izquierdos y 1 fragmento de calcáneo (MC-5), falange (superficie), 3 fragmentos de cráneo (sin rótulo) y 1 molar suelto (sin rótulo) (Fig. 5). Además se recuperaron 11 fragmentos que no pudieron ser identificados.

Todos estos restos corresponden a la especie humana y por sus estados de fusión pertenecen a individuos subadultos. Sin embargo no pudieron ser individualizados, es decir si corresponden al individuo 2 o 3 y tampoco se descarta la posibilidad de que perteneciera a otro individuo ya que todos los restos se encontraron en un conjunto mezclado.

\section{Discusión y consideraciones finales}

Las diferentes intervenciones y técnicas de extracción, llevaron a un registro desorganizado y descontextualizado reduciendo las posibilidades de obtención de información. Las tareas de rescate llevadas a cabo por los autores, permitieron recuperar una mínima porción de las partes esqueletarias aún enterradas. Los procedimientos realizados por bomberos y policía local, produjeron una pérdida de la relación espacial de las unidades anatómicas correspondientes a cada uno de los distintos individuos recuperados, además de pérdidas en el proceso de intermediación en el transporte y guardado. Otro factor importante fue la perdida de especímenes por acción del mar, verificada entre las visitas realizadas al sitio (Fig. 6). Sin embargo, se pueden plantear algunas hipótesis sobre las prácticas de inhumación.

La información obtenida en este sitio incrementa el conocimiento bioantropológico regional y permite discutir nuevas preguntas. Las evidencias presentadas conforman los primeros datos sobre prácticas mortuorias en la costa marina de Pampa Deprimida para el Holoceno tardío. Dentro de la región, el sitio Laguna La Colorada (3400 AP) se localiza en las llanuras continentales; aún cuando no se cuenta con información acerca de la disposición de los restos, las inhumaciones se recuperaron en un sector aislado a unos 300m del área de actividades registrado (Aldazabal y Cáceres, 1998). En el sitio La Sa- 

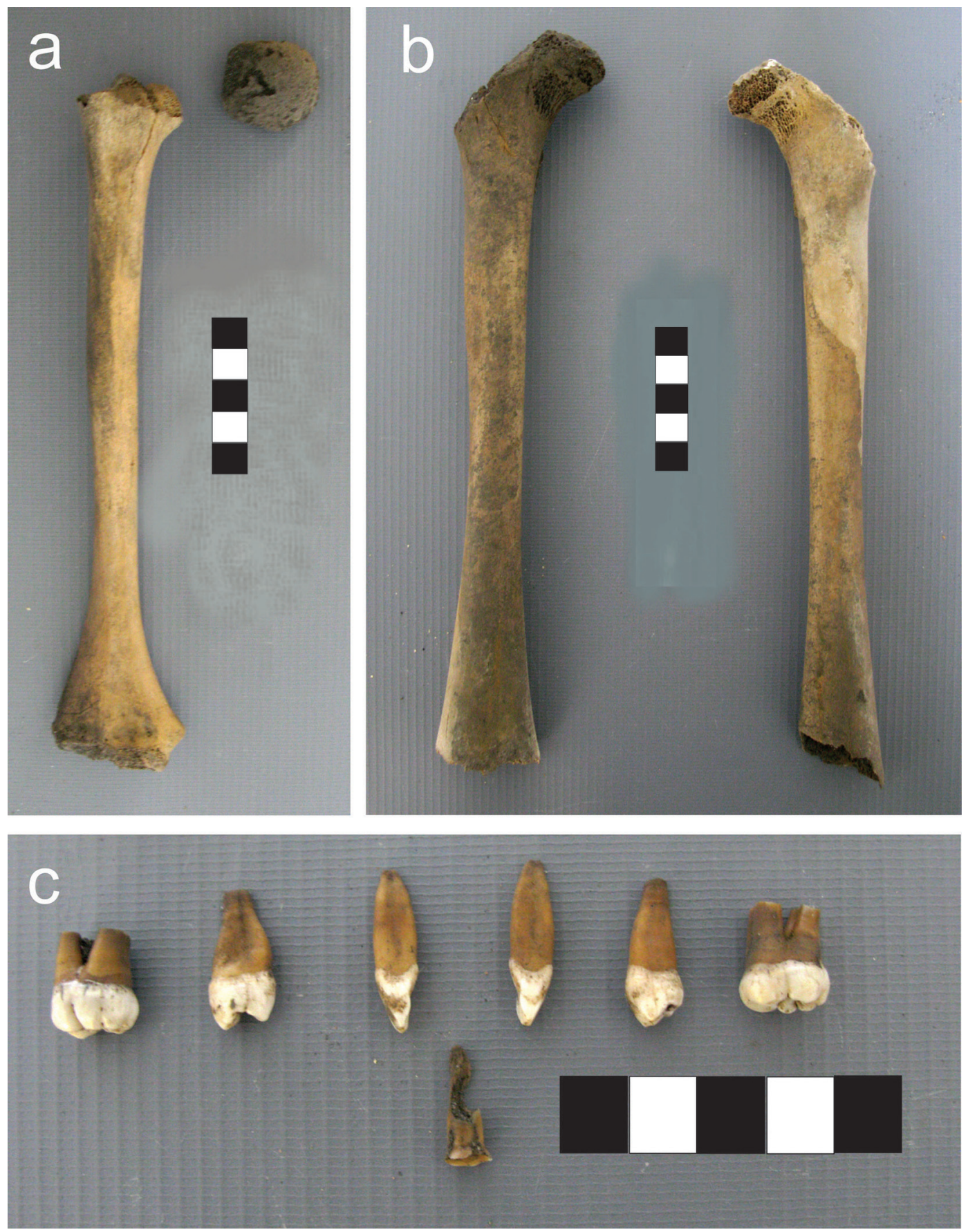

Fig. 4. Individuo 3. a) Húmero derecho. b) Fémures derecho e izquierdo. c) Piezas dentales.

lada, con una cronología en el Holoceno tardío (1420 AP), se registraron varios individuos de edad adulta y subadultos, en disposición paralela a la costa de la laguna, también a cierta distancia del área de actividades (Aldazabal,
1993) y los restos de laguna Sotelo (circa 1000 AP), distantes sólo $6 \mathrm{~km}$ del conjunto en estudio, comprenden algunas piezas aisladas dentro del área de asentamiento (Eugenio y Aldazabal, 1987-88; Murgo y Aldazabal, 2007). 

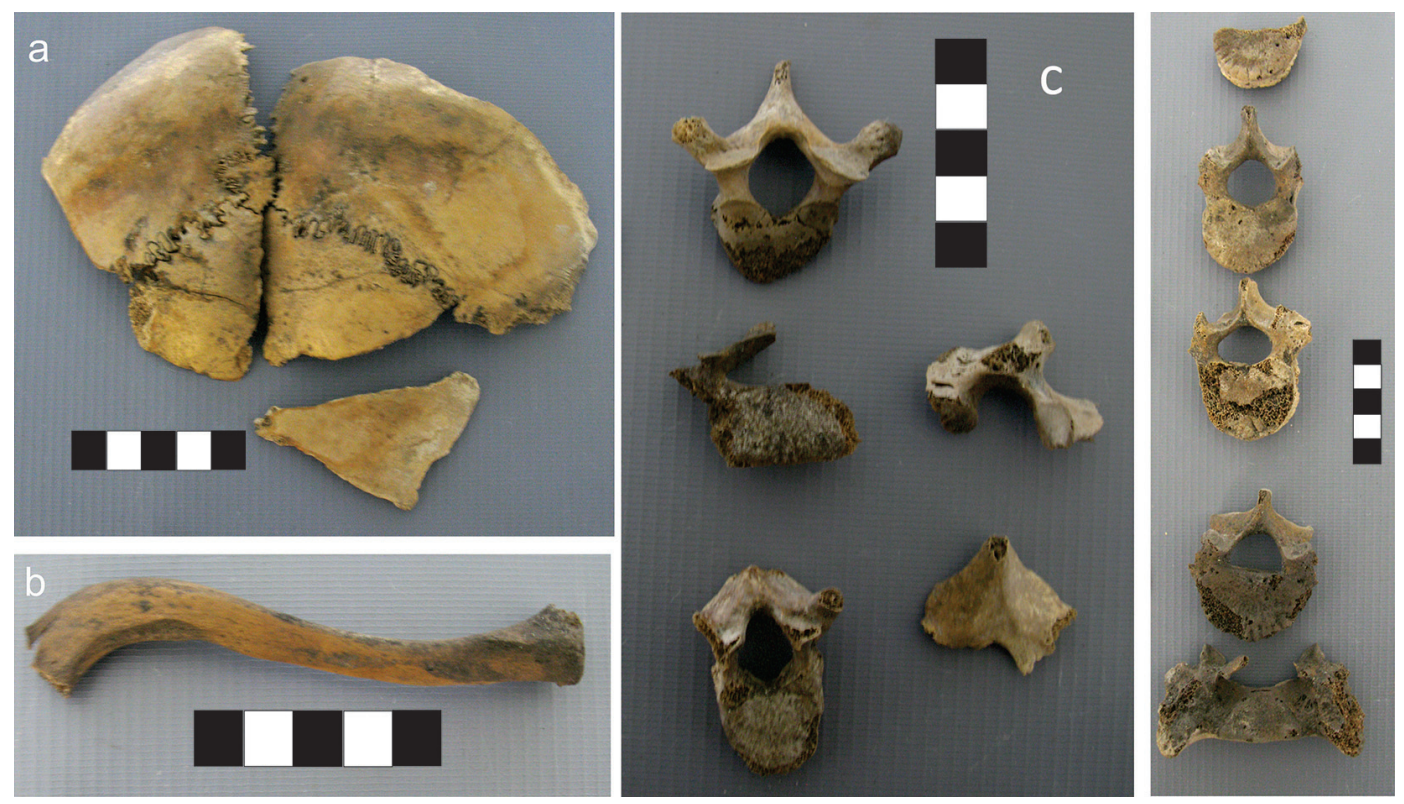

Fig 5. Restos sin individualizar. a) Fragmentos de cráneo. b) Clavícula. c) Vértebras.

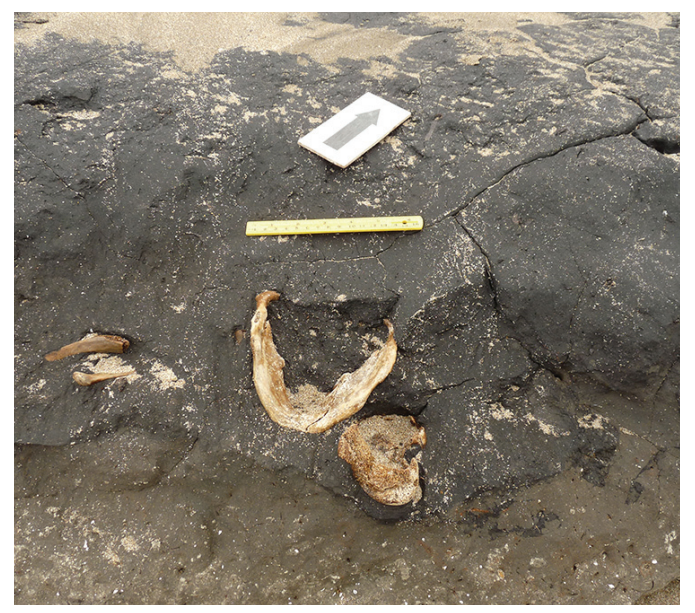

Fig. 6. Remoción por erosión marina.

Respecto a la preservación de los restos, y en relación a posibles procesos postdepositacionales, el lugar de hallazgo es de escasa circulación (aún cuando, estando prohibido, por ese sector pasan ocasionalmente vehículos $4 \times 4$ ), lo que favoreció que los especímenes se encontraran en buen estado de completitud, con un bajo nivel de fractura. Los restos no presentaban rastros de carbonatación, ni de óxidos de manganeso, así como tampoco signos de meteorización o blanqueamiento diferencial, características que sugieren un enterramiento primario y que solo fueron expuestos en ocasión del hallazgo. Los entierros primarios son los que caracterizan con más frecuencia los sitios conocidos para el Holoceno tardío en Pampa y Nordpatagonia oriental. Además la localización de las inhumaciones en un paisaje medanoso es también una constante en la costa sur de la provincia de Buenos Aires y norte de Patagonia (Bonomo, Scabuzzo y Leon, 2013; Eugenio y Aldazabal, 2004; Martínez y Flensborg, 2018; entre otros).

La existencia de por lo menos tres o cuatro individuos sin evidencia de otras actividades en el lugar, sugiere una selección del lugar como especifica de entierro probablemente relacionada con una valoración especial de este paisaje (Carr, 1995). En este sentido también aporta el valor $\delta^{13} \mathrm{C}$ : $20 \pm 2 \%$ informado por el laboratorio, obtenido sobre colágeno del fémur fechado, que estaría señalando una dieta mayormente continental, indicando un lugar de residencia hacia el interior de los médanos, en localizaciones probablemente similares al sitio Laguna Sotelo.

Los relevamientos efectuados junto con la información y las fotografías facilitadas por EAAF permiten establecer que la distribución de los restos cubre un espacio de $3 \mathrm{~m}^{2}$. El registro de la mayor parte de las unidades anatómicas y la ausencia de huellas de procesamiento su- 
giere que se trata de entierros primarios de individuos completos. La distribución y orientación de las unidades anatómicas observadas en el terreno y registradas fotográficamente sugieren además, un entierro primario con los miembros inferiores flexionados por lo menos en un individuo (Fig. 7).

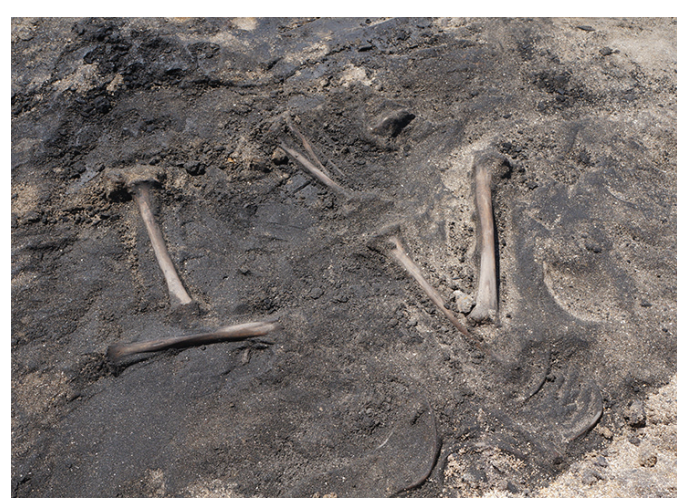

Fig. 7. Disposición flexionada.

Por otra parte, es de destacar la presencia de individuos de diferentes edades y subadultos pequeños, hecho que sólo se observó en el sitio La Salada (Aldazabal, 1993). El hallazgo de tres artefactos junto a los individuos 1-2 podría ser interpretado como un descarte o pérdida casual, o haber sido intencionalmente dejados como ajuar. Análisis arqueométricos en proceso permitirán ampliar y complementar nuestras inferencias.

\section{LITERATURA CITADA}

Aldazabal, V. (1993). Análisis morfológico de los restos humanos provenientes del sitio arqueológico La Salada, Pdo. de Castelli, Pcia. Buenos Aires. Arqueología, 3, 155-170

Aldazabal, V. y Bellotti, C. (2019). Guanacos en la playa. Revista de Antropología del Museo de Entre Ríos 5(1), 15-23

Aldazabal, E. y Cáceres, L. (1998). Primeras observaciones en el sitio arqueológico La Colorada, Pdo. de Rauch. En: Actas del XII Congreso Nacional de Arqueología Argentina, Tomo 3, pp. 95-102, Universidad Nacional de La Plata, La Plata.

Bonomo M., Scabuzzo, C y Leon, D. C. (2013). Cronología y dieta en la costa atlántica pampeana, Argentina. Intersecciones en Antropología 14, 123-136.
Buisktra J. y Ubelaker, D. (1994). Standards for data collection from human skeletal remains. Arkansas, Estados Unidos: Archeological Survey Research Series No. 44.

Carr, C. (1995). Mortuary practices: Their social, philosophical-religious, circumstantial, and physical determinants. Journal of Archaelogical Method and Theory 2(2), 105-200. doi:10.1007/BF02228990

Eugenio, E. y Aldazabal V. (1987-88). El sitio arqueológico laguna de Sotelo, Partido de Mar Chiquita, provincia de Buenos Aires. Paleoetnologica 4, 79-86.

Eugenio, E. y Aldazabal V. (2004). Los cazadores recolectores del litoral marítimo del área de Bahía de San Blas, provincia de Buenos Aires. En Civalero, M. T., Fernández, P. y Guráieb, G. (Comps.). Contra viento y marea. Arqueología de Patagonia (pp. 687-700). Buenos Aires, Argentina: INAPL.

Eugenio, E. y Aldazabal, V. (2017). Rescate arqueológico en la costa marina del Partido de Mar Chiquita. Trabajo presentado a las $2^{\circ}$ Jornadas Bonaerenses de Conservación Costera. Ms.

Fazekas, I. y Kósa, F. (1978). Forensic fetal osteology. Budapest, Hungría:Akadémiai Kiadó.

García Laborde, P. (2018). Informe de restos óseos humanos. Sitio Mar Chiquita 1. Mar Chiquita. Buenos Aires. Ms.

Guichón, R. y Garcia Laborde, P. (2014). Determinación sexo etaria en individuos subadultos. Análisis preliminar en la Misión salesiana de Rio Grande, Tierra del Fuego. En Castro Esnal, A., Funes, M. L., Grosso, M., Kuperszmit, N., Murgo, A. yRomero, G. (Eds.). Entre pasados y Presentes IV (pp. 391-407). Buenos Aires, Argentina: INAPL.

Krenzer, U. (2006). Compendio de métodos antropológico forenses. Serie de Antropología Forense. Tomo 2. Métodos para la determinación de sexo. Guatemala, Guatemala: CAFCA.

Martínez, G. y Flensborg, G. (2018).Nuevos contextos funerarios en la transición Pampeano-Patagónica Oriental (Pcia. de Buenos Aires, Argentina): Aportes al Holoceno medio y tardío inicial. Chúngara Revista de Antropología Chilena 50(3), 441-458. doi:10.4067/S071773562018005001101

Murgo, A. y Aldázabal, V. (2007). Análisis de isótopos estables de carbono y nitrógeno de los restos óseos humanos provenientes de sitios área Pampa Deprimida centro oriental. En: Oliva, F., de Grandis N. y Rodríguez, J. (Eds.). Arqueología Argentina en los inicios de un nuevo siglo (pp. 259-264). Rosario, Argentina: Universidad Nacional de Rosario.

Schaefer, M. S., Black, M. C., Schaefer, C. y Scheuer, L. (2009). Juvenile osteology. San Diego, Estados Unidos: Elsevier Academic Press. doi :10.1016/B978-0-12374635-1.X0001-X

Scheuer, L. y Black, S. (2000). Development and ageing of the juvenile skeleton. London, UK: Greenwich Medical Media.

https://epdf.pub/standards-for-data-collection-fromhuman-skeletal-remains-proceedings-of-a-semin.html. doi:10.1016/B978-012624000-9/50003-4

Schutkowski, H. (1993). Sex determination of infant and juvenile skeletons: I. Morphognostic features. American Journal of Physical Anthropology 90, 199-205. doi:10.1002/ajpa.1330900206 